\title{
Wave particle interactions in the high-altitude polar cusp: a Cluster case study
}

\author{
B. Grison ${ }^{1}$, F. Sahraoui ${ }^{1}$, B. Lavraud ${ }^{2}$, T. Chust ${ }^{1}$, N. Cornilleau-Wehrlin ${ }^{1}$, H. Rème ${ }^{3}$, A. Balogh ${ }^{4}$, and M. André ${ }^{5}$ \\ ${ }^{1}$ Centre d'étude des Environnements Terrestre et Planétaires, 10/12 avenue de l'Europe, 78140, Vélizy, France \\ ${ }^{2}$ Space Science and Applications Los Alamos National Laboratory, MS D466, Los Alamos, NM 87545, USA \\ ${ }^{3}$ Centre d'Etude Spatiale des Rayonnements, 9 avenue du Colonel, 31028 Toulouse Cedex 4, France \\ ${ }^{4}$ Space and Atmospheric Group, The Blackett laboratory, Imperial College, Prince Consort road, London, UK \\ ${ }^{4}$ Swedish Institute of Space Physics, Uppsala, Sweden
}

Received: 21 December 2004 - Revised: 27 October 2005 - Accepted: 28 October 2005 - Published: 23 December 2005

\begin{abstract}
On 23 March 2002, the four Cluster spacecraft crossed in close configuration ( $\sim 100 \mathrm{~km}$ separation) the high-altitude $\left(10 R_{E}\right)$ cusp region. During a large part of the crossing, the STAFF and EFW instruments have detected strong electromagnetic wave activity at low frequencies, especially when intense field-aligned proton fluxes were detected by the CIS/HIA instrument. In all likelihood, such fluxes correspond to newly-reconnected field lines. A focus on one of these ion injection periods highlights the interaction between waves and protons. The wave activity has been investigated using the $\mathrm{k}$-filtering technique. Experimental dispersion relations have been built in the plasma frame for the two most energetic wave modes. Results show that kinetic Alfvén waves dominate the electromagnetic wave spectrum up to $1 \mathrm{~Hz}$ (in the spacecraft frame). Above $0.8 \mathrm{~Hz}$, intense Bernstein waves are also observed. The close simultaneity observed between the wave and particle events is discussed as an evidence for local wave generation. A mechanism based on current instabilities is consistent with the observations of the kinetic Alfvén waves. A weak ion heating along the recently-opened field lines is also suggested from the examination of the ion distribution functions. During an injection event, a large plasma convection motion, indicative of a reconnection site location, is shown to be consistent with the velocity perturbation induced by the large-scale Alfvén wave simultaneously detected.
\end{abstract}

Keywords. Magnetospheric physics (Magnetopause, Cusp and boundary layers; Plasma waves and instabilities) - Space plasma physics (Wave-particle interactions)

\section{Introduction}

The cusps, supposedly located on open magnetic field lines, are key regions for transfer of mass and momentum from

Correspondence to: B. Grison

(benjamin.grison@cetp.ipsl.fr) the magnetosheath to the Earth's magnetosphere. Solar wind particles can directly access to the ionosphere through it. The magnetic reconnection process in an open magnetosphere model creates newly-opened field lines that are populated with magnetosheath ions and convected across this region under southward (Dungey, 1961) or northward (Dungey, 1963) IMF (Interplanetary Magnetic Field). The high-altitude cusp is adjacent to the magnetosheath and thus closer to reconnection sites than lower altitude cusp regions. After a few crossings by the HEOS spacecraft, providing a first description of the high-altitude cusp (Paschmann et al., 1976), Gurnett and Frank (1978), using Hawkeye 1 data, highlighted that magnetic fluctuations in the ultra-lowfrequency range are an indicator of this region. The POLAR spacecraft, with its highly elliptical orbit, first surveyed this region up to $9 R_{E}$ (Earth radii) in detail. From this spacecraft data, various kinds of waves have been identified in this region, such as whistler waves and sinusoidal bursts in the low frequency range (Pickett et al., 2001). This activity occurs on a broad-band scale and similar spectral properties are found in the high-altitude cusp as in the surrounding regions, such as the low-latitude boundary layer (Tsurutani et al., 1998). In the ULF range, electromagnetic waves around the local proton cyclotron frequency with highly variable properties are seen in and around this cusp region (Le et al., 2001). This wave activity has been linked with two major processes: strong field-aligned flows of particles and counterstreaming ions (Le et al., 2001). The first one could correspond to ion injections from magnetic reconnection sites. The specific distribution functions formed at such locations (Cowley, 1982) can be a source of free-energy which could explain wave generation (Bingham et al., 1999) along the newlyreconnected field lines. The second process, counterstreaming ions, is expected on open field lines some time after reconnection and is favourable to wave apparition (Nykyri et al., 2003). Pickett et al. (2001) suggested that part of the wave activity observed in the high-altitude cusp region could be generated at the reconnection site, too. In particular, 
the Alfvénic perturbations that originate from the reconnection site (Haerendel et al., 1978) could propagate along the newly-opened flux tube into this region.

The Cluster mission permits one to explore extensively this high-altitude cusp region with the unique opportunity given by the four spacecraft to decouple spatial and temporal effects. Nykyri et al. (2003) found clear peaks around the local proton gyrofrequency without any correlation between spacecraft signals while separated by $600 \mathrm{~km}$. They concluded that waves are generated locally due to sheared plasma flows. With a study on the direction of the Poynting flux below and above the proton gyrofrequency, Sundkvist et al. (2005) also concluded that there is local wave generation along the field lines.

At low altitudes, in the cusp as in the nearby auroral region, wave and particle interactions have been frequently studied (e.g. Bouhram et al., 2003). Ion beams (Cattell et al., 2002), temperature anisotropy (André et al., 1986) and velocity shears (Lakhina, 1990) are plasma instabilities commonly observed. Bernstein modes and Alfvén modes obviously interact with particles in this region (Kintner et al., 1986; Chaston et al., 2004). Broad-band extremely lowfrequency (BBELF) waves - observed with Alfvén waves are associated with the most common types of oxygen heating (André et al., 1998). Electromagnetic waves in the top ionosphere are often told to originate from higher altitudes (e.g. Le Queau and Roux, 1992). This idea is partly confirmed by the simultaneous observations of alfvénic electromagnetic activity in the high-altitude cusp region and of ionospheric convection (Farrugia et al., 2004).

Here we study the cusp crossing that occurred on 23 March 2002 when the Cluster fleet was in close configuration in the cusp region (100 km of intercraft separation). After introducing instrumentation and interplanetary magnetic conditions, we present a general overview of this crossing (Sect. 2). In Sect. 3, we discuss the correlation between strong ion injection events and ULF magnetic activity. A focus on one of these events highlights these correlations and suggests that wave-particle interaction takes place in the cusp. Then we present in Sect. 4 the wave mode identification by means of the k-filtering analysis. Finally, we discuss our results, in order to explain the presence and origin of these modes.

\section{Observations}

\subsection{Instrumentation}

The plasma wave and particle data presented below have been measured by several instruments on board Cluster. Three magnetic components of the waves are measured by the Spatio Temporal Analysis of Field Fluctuations (STAFF) experiment (Cornilleau-Wehrlin et al., 2003). Two electric components are measured in the spin plane by the Electric Field Wave (EFW) experiment (Gustafsson et al., 2001). These two instruments provide for this study waveform data at a sampling rate of $25 \mathrm{~Hz}$. Let us call the STAFF waveform data the STAFF-SC data. The three-axis search coil magnetometer that constitutes part of the STAFF experiment is not able to measure fluctuations at frequencies lower than about $0.1 \mathrm{~Hz}$. Also, a Doppler shift of $\pm 0.25 \mathrm{~Hz}$, due to the 4-s spin of the spacecraft, distorts the magnetic components measured in the plane perpendicular to the spin axis. STAFF-SC measurements of low frequency magnetic field fluctuations (frequencies lower than about $0.35 \mathrm{~Hz}$ ) are thus not accurate. The low frequency counterpart of the magnetic fluctuations are provided by the Flux Gate Magnetometer experiment (FGM) (Balogh et al., 2001). The CIS instruments (Rème et al., 2001) measure full 3-D ion distribution functions and moments (density, bulk flow velocity, temperature) at a time resolution up to the spin period. In this paper, we use the Hot Ion Analyser (HIA) data, which does not resolve ion species but has high energy and angular resolution. The IMF is provided by the ACE spacecraft that orbits at the L1 libration point.

\subsection{Orbit}

On 23 March 2002 the Cluster spacecraft crossed the highaltitude cusp from the northern lobe to the magnetosheath. This cusp crossing has also been studied by Lavraud et al. (2004). The outbound trajectory, approximately in the midnight-noon magnetic meridian, was mainly sunward. Figure 1 shows the Cluster spacecraft 1 orbit from 09:00 to 12:30 UT (black marks), with magnetospheric field lines derived from the T89 model (Tsyganenko, 1989). The spacecraft tetrahedron projection in the noon-midnight plane is shown at 09:00 and 12:30 UT. The intercraft separations during the crossing were about $100 \mathrm{~km}$ and, for the sake of visibility, are enlarged by a factor of 100 in Fig. 1.

\subsection{Interplanetary conditions}

Figure 2 displays the IMF measured by ACE. The IMF clock angle, the three GSM components, and the IMF modulus are, respectively, plotted from bottom to top. The data have been shifted by 57 min forward to account for the solar wind convection time from ACE to the Cluster location. This shift is estimated with the mean bulk antisunward velocity in the considered time interval and before 11:50 UT (shifted time on Fig. 2). At this time, there is a strong and abrupt increase in the IMF (up to $12 \mathrm{nT}$ ), associated with a sudden enhancement of the solar wind density and bulk velocity, which could be a signature of an interplanetary shock. This shock is seen later in the FGM data, around noon (not shown), when Cluster has already reached the magnetosheath. This is why the time delay has been computed in averaging velocities before that shock. The same shift is used during the crossing.

Before the shock the field magnitude is almost constant (around 4 or $5 \mathrm{nT}$ ). The IMF is northward, except during three intervals: between 10:37 and 10:47 UT, between 10:51 and 11:01 UT and between 11:38 and 11:51 UT. Until 10:35 UT the clock angle is less than $30 \mathrm{deg}$, which means the $B_{z}$ component dominates during this interval. The $B_{y}$ 


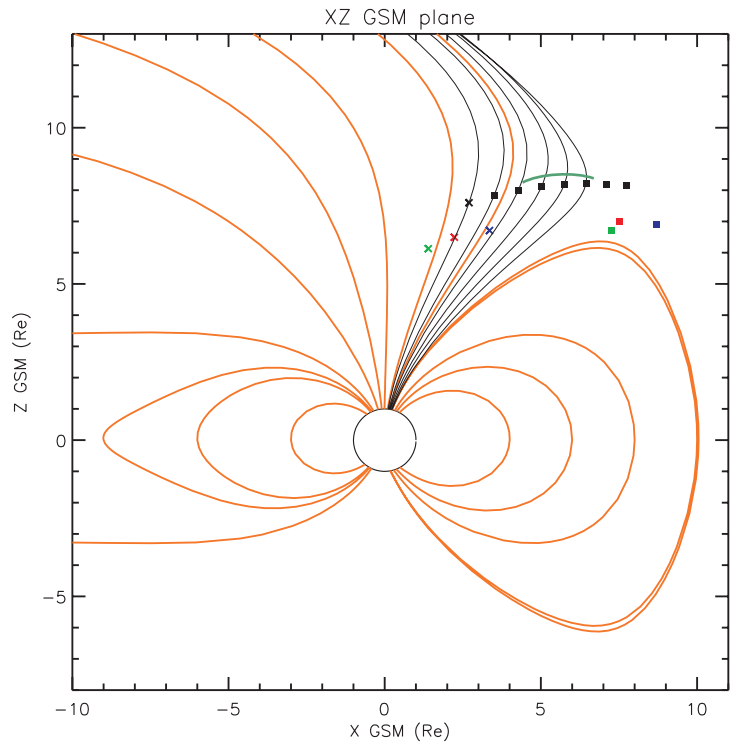

Fig. 1. Cluster spacecraft orbit between 09:00 and 12:30 UT on 23 March 2002, projected on the $X_{g s m}, Z_{g s m}$ plane. The field lines have been computed from the T89 model. The yellow lines are the inner magnetosphere lines at 11:40 UT, whereas the black ones are the field lines that are going through the SC1 (black marks) position every $30 \mathrm{~min}$. The constellation, following the Black-RedGreen-Blue (BRGB) colour code (respectively, for spacecraft 1, 2, 3 and 4), has been plotted at 09:00 (BRGB crosses) and at 12:30 UT (BRGB squares). The inter-spacecraft separation centred around $\mathrm{SC} 1$ has been enlarged one hundred times. The green line indicates the part of the Cluster orbit that has been identified in the cusp.

is really dawnward (negative), between 10:30 and 11:15 UT, and after 11:32 UT. Otherwise $\left|B_{y}\right|$ remains low (less than $2 \mathrm{nT})$. The $B_{x}$ component fluctuates between -2 and $2 \mathrm{nT}$ until 11:02 UT. Then it becomes directed sunward (positive). It is worth noticing that the reconnection process at antiparallel merging sites is likely to occur in the lobes when the IMF is northward, i.e. during the major part of crossing. As long as the clock angle is close to $0 \mathrm{deg}$, mainly until 10:37 UT, the anti-parallel merging sites are expected to be located poleward of the spacecraft.

\subsection{Overview of the Cluster data}

Figure 3 displays an overview of the wave and ion data obtained by SC1 during the 10:00-11:54 UT interval. From top to bottom, one sees, the ion plasma density (HIA) (red curve, panel a) and the magnetic field intensity (FGM) (black curve, panel a), the Alfvén velocity (red curve, panel b) and the $\delta E / \delta B$ ratio (black curve, panel b) derived from the power of the fluctuations measured by STAFF-SC and EFW over the range $0.35-2 \mathrm{~Hz}$, the energy spectra of the ions (HIA) (panel c), the parallel (black) and perpendicular (red) components, with respect to the magnetic field (as all following references to parallel or perpendicular component), of the bulk flow velocity (HIA) (panel d), the power of the magnetic field fluctuations averaged over its three components

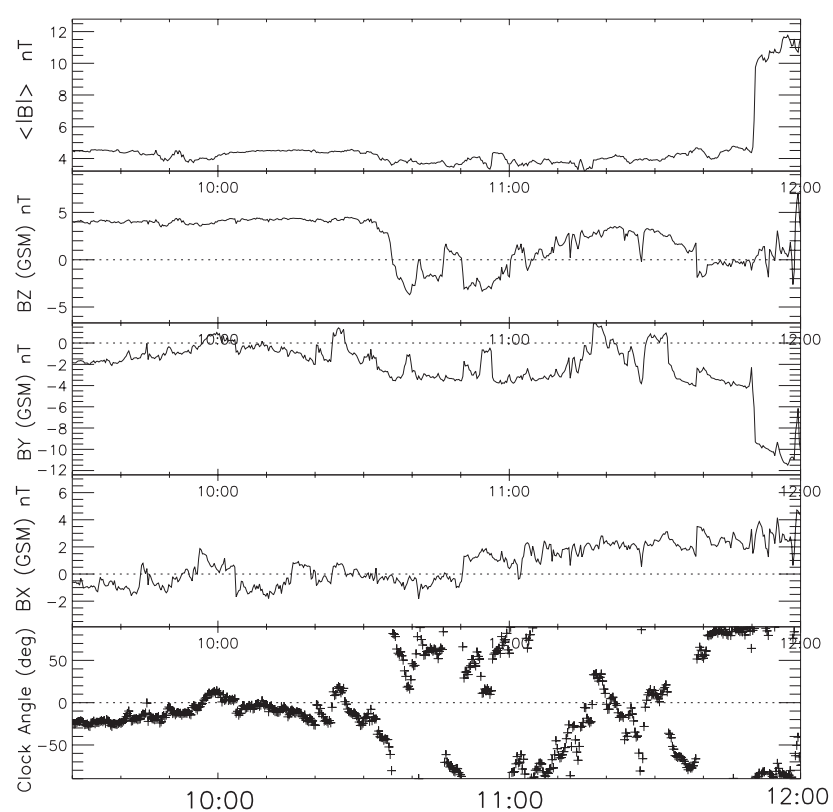

Fig. 2. Lagged IMF measured by ACE spacecraft. From top to bottom, in GSM (Geocentric Solar Magnetospheric) coordinate system, the solar magnetic field magnitude, the $x, y$ and $z$ magnetic components and the clock angle $\left(\arctan \left(B_{y} / B_{z}\right)\right)$ are displayed between 09:30 and 12:00 UT. The calculated delay between ACE and Cluster is $57 \mathrm{~min}$.

and integrated over the $1-10 \mathrm{~Hz}$ frequency range (STAFFSC) (panel e), the dynamic power spectra of the magnetic field fluctuations derived from the STAFF-SC data (FFT have been performed on intervals of 512 points, giving an about 20-s time resolution of the dynamic spectra) (panel $\mathrm{f}$ ), the pitch angle spectra of the ions (HIA) (panel g) and the dynamic power spectra of the electric field (EFW) (panel h). Because of the lack of EFW data on board SC1, we use those on board SC4. The comparison between these two data sets is relevant at a time resolution of about $20 \mathrm{~s}$. Indeed, the comparison of the FGM data between the four spacecraft, on the time scale of $20 \mathrm{~s}$, shows almost identical fluctuations, as the spacecraft are separated by only $100 \mathrm{~km}$.

First, the spacecraft are possibly in the high-latitude boundary layer (HLBL), which is characterized by a very small plasma density but magnetosheath-like ions. At 10:10 UT, the spacecraft enter in the distant polar cusp where a strong magnetic depression (down to $15 \mathrm{nT}$, panel a) and magnetosheath-like ions are detected (distribution centred around $800 \mathrm{eV}$, panel c). The magnetic field (panel a) displays high levels of typical cusp fluctuations between 10:45 and 11:20 UT. The ion flux and the density (respectively, panels $\mathrm{c}$ and a) progressively increase in the cusp until 10:16 UT. Then they remain almost constant. The parallel flow velocity (panel d) is mainly directed earthward (positive) until 10:55 UT. Additional observations can be made from the inspection of the pitch angles of the ions during this period (panel g): one can notice the alternation of two distinct 
CLUSTER SC1 23/March/2002

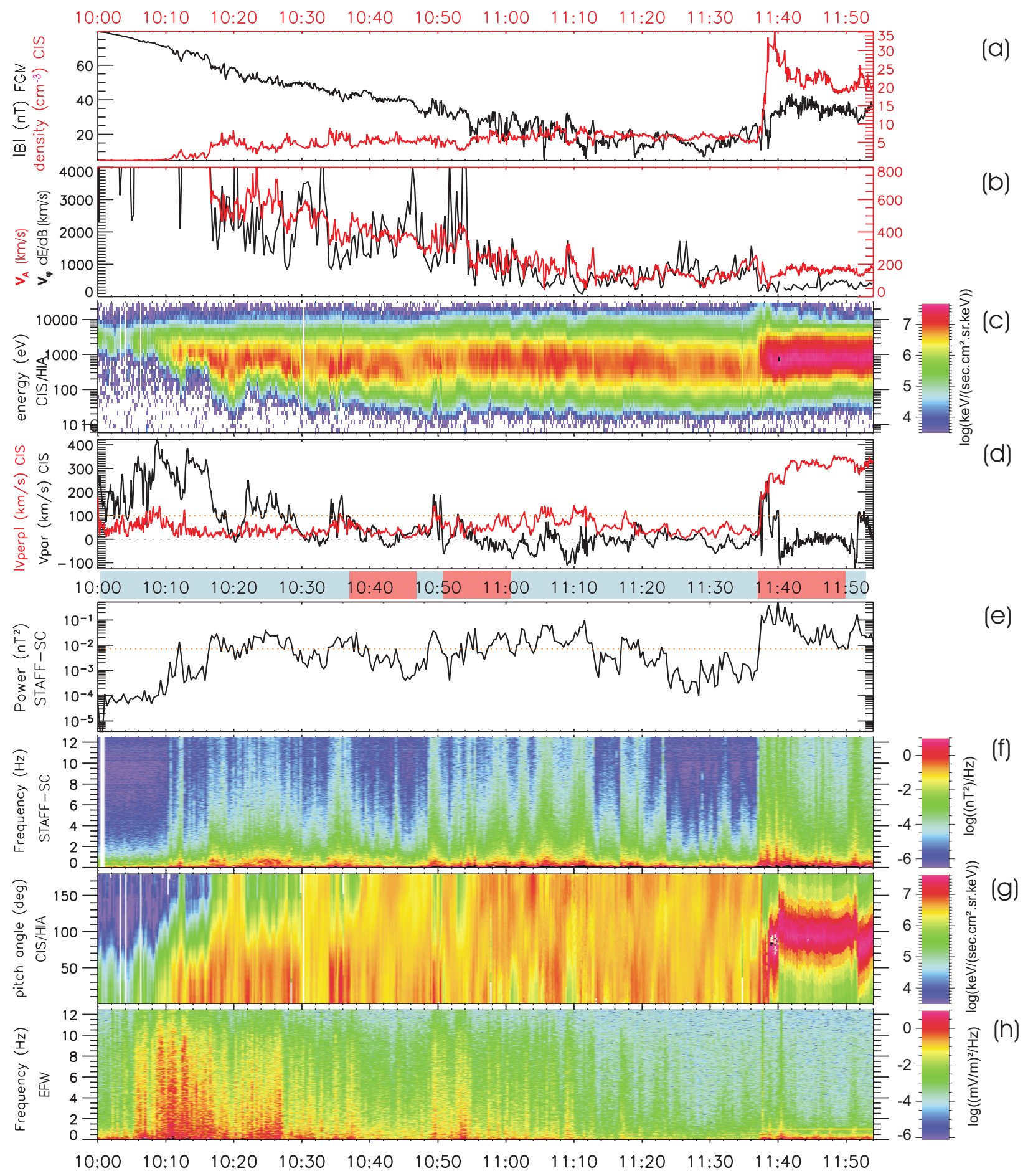

Fig. 3. Overview of the cusp crossing on 23 March 2002. The data are provided from various instruments on board spacecraft 1, except EFW, whose data are taken from SC4. Panel (a) displays the DC magnetic field given by FGM (black curve) and the density derived from HIA (red curve). Panel (b) displays the $\delta E / \delta B$ ratio (black curve) and the Alfvén velocity derived from HIA and FGM data (red curve). Panel (c) displays the ion energy spectrogram (HIA), (d) the parallel component of the bulk flow velocity (HIA) (black curve) and the norm of perpendicular component (red curve). Panel (e) shows the power of the magnetic field fluctuations integrated between 1 and $10 \mathrm{~Hz}$ (STAFF$\mathrm{SC}$ ), (f) the time frequency spectrogram of these fluctuations (STAFF-SC), (g) the ion pitch angle spectrogram (pitch angles are calculated in the spacecraft frame over the $[100,1000] \mathrm{eV}$ energy range) (HIA) and (h) the time-frequency spectrogram of electric field fluctuations (EFW). Time interval between those panels is coloured in blue (red) for a northward (southward) IMF. 
regions, a region being characterized by a strongly asymmetric distribution of the pitch angles with ions moving mainly earthward (low pitch angles) and another region being characterized by a fairly isotropic pitch angle distribution of the ions. The parallel velocity (panel d) turns to negative from 10:55 UT until 11:20 UT. Pitch angle distribution during this period confirms the dominance of ion outflow. Fluctuations of the perpendicular and parallel components of the flow velocity (panel d) are of the same intensity. After 11:20 and until 11:38 UT, both velocity components are less than $50 \mathrm{~km} / \mathrm{s}$, corresponding to a stagnation region (Lavraud et al., 2004). Concerning the wave activity, one observes that magnetic fluctuations (panels e and f) start at about 10:10 UT with bursts of various intensity and duration. The mean wave activity level increases after 11:38 UT. The maximum of electric fluctuations (panel h) is reached at about 10:12 UT. Then their intensity decreases. One can notice localized enhancements around 10:54 and 11:40 UT and a very low activity between 11:10 and 11:38 UT. From panel (b) one could notice that the $\delta E / \delta B$ ratio and the Alfvén velocity have a similar trend. A very large $\delta E / \delta B$ ratio observed at 10:33, 10:46, 10:52 UT and 10:54 UT suggests an electrostatic wave activity. However, except at 10:54 UT, these cases are associated neither with strong electric field fluctuations nor with magnetic fluctuations, electrostatic wave activity being thus very weak: the wave activity observed in the cusp is essentially electromagnetic. So, as already noticed by André et al. (2001) during another crossing, the $\delta E / \delta B$ ratio varies in relationship with background parameters, in this instance the magnetic field and the density. It can be seen, in particular, from the electric fluctuations (panel h), the trend of the $\delta E / \delta B$ ratio of the wave activity is correlated with the largescale decrease of the background magnetic field intensity.

On each panel, a clear boundary, the magnetopause, is encountered at about 11:38 UT (see Lavraud et al. (2004) for a description of this boundary). The mean energy of the particles is roughly the same but their density (intensity of the flux) clearly increases at this boundary. Between 11:38 and 11:40 UT the bulk velocity changes abruptly because the ions flow approximately perpendicular to the magnetic field at about $300 \mathrm{~km} / \mathrm{s}$. The magnetopause encounter is very dynamic and appears to be correlated with the arrival of the interplanetary shock, as previously discussed. In summary, in the cusp part of this event (10:10-11:38 UT), which is the main purpose of this paper, the ion flow velocity highlights three successive regions crossed by the spacecraft. From about 10:10 UT to 10:55 UT, the bulk flow velocity is dominated by injections of magnetosheath ions and their relaxation to an equilibrium distribution. Then, until 11:20 UT, the plasma is characterized by an enhancement of the convection and the predominance of mirroring ions (when negative parallel velocity peaks are detected). Finally, and until the magnetopause crossing, the plasma almost stagnates with a flow velocity smaller than $50 \mathrm{~km} / \mathrm{s}$ (see Lavraud et al. (2002) for a detailed study of such region). We will now deal with the relationship between the ions and ULF waves.

\section{Correlation between wave and particle events}

\subsection{Correlation over the whole cusp crossing}

Let us first examine the ULF power, on panel (e) of Fig. 3. To do so, we fix $7.10^{-3} \mathrm{nT}^{2}$ as the minimal threshold (see dotted yellow line on panel e) for considering wave activity as significant: it corresponds roughly to a mean level of the wave power. The first time the power exceeds this value happens around 10:12 UT for a short duration. Later, it occurs during time intervals longer than 2 min: between 10:16 and 10:19 UT, between 10:22 and 10:29 UT and between 10:34 and 10:38 UT. Then peaks are shorter ( $2 \mathrm{~min})$ and are centred around 10:50, 10:55, 10:59, 11:02, 11:06, 11:11 and 11:18 UT. We recall that all the magnetic fluctuations identified above are of an electromagnetic nature (finite $\delta E / \delta B$ ratio).

On panel (d), the ion perpendicular flow velocity varies between 0 and $150 \mathrm{~km} / \mathrm{s}$, in a relatively smooth way, while the parallel flow velocity fluctuates much more abruptly. In the same way as we did for inspecting the wave power, we define a $100 \mathrm{~km} / \mathrm{s}$ threshold for identifying the intervals when flow velocities, either parallel or perpendicular to the magnetic field, are large. At the entrance into the cusp (from 10:10 to $10: 16 \mathrm{UT}$ ), where the plasma density is low, very high velocities (up to $400 \mathrm{~km} / \mathrm{s}$ ) are observed. Later, the periods of large velocities correspond to much shorter time intervals at 10:18, 10:22, 10:23, 10:25, 10:26, 10:28, 10:34, 10:36, 10:49, 10:50 and 10:53 UT. All these intervals correspond to positive parallel velocities, meaning earthward flows. Large, negative (upgoing), parallel components of the flow velocity are detected later on at 11:01, 11:04 and 11:09 UT. In conjunction with a large value of the parallel flow velocity, the perpendicular flow velocity also reaches large values (at least $100 \mathrm{~km} / \mathrm{s}$ ) at 10:08, 10:09, 10:13, 10:35 and 10:50 UT. Then, but with low values of the parallel flow velocity, this high level of the perpendicular flow velocity is again observed at 10:59, 11:03, 11:18 UT and for longer time intervals (about $2 \mathrm{~min}$ ) around 11:06 and 11:11 UT.

From these observations, one notices that the sharper the fluctuations of the bulk flow velocity are, the more intense the associated electromagnetic wave activity. During the first part of the crossing (before 10:53 UT), injections of magnetosheath ions (enhanced earthward flows of field-aligned ions) occur when the IMF is northward and are associated with a strong ULF electromagnetic activity. Until 10:44 UT the convection motion is sunward (not shown). Since the spacecraft are on the lobe side of the cusp, these observations are consistent with one or more reconnection sites situated tailward and above the spacecraft (e.g. Vontrat-Reberac et al., 2003). In addition, two long periods without injections (10:38-10:48 UT and 10:51-11:01 UT) are observed when the IMF is southward. The spacecraft location and the fact that reconnection takes place very probably in the subsolar region of the magnetosphere during a southward IMF period (e.g. Smith and Lockwood, 1996) may explain the nonobservation of injection signatures during these intervals. 


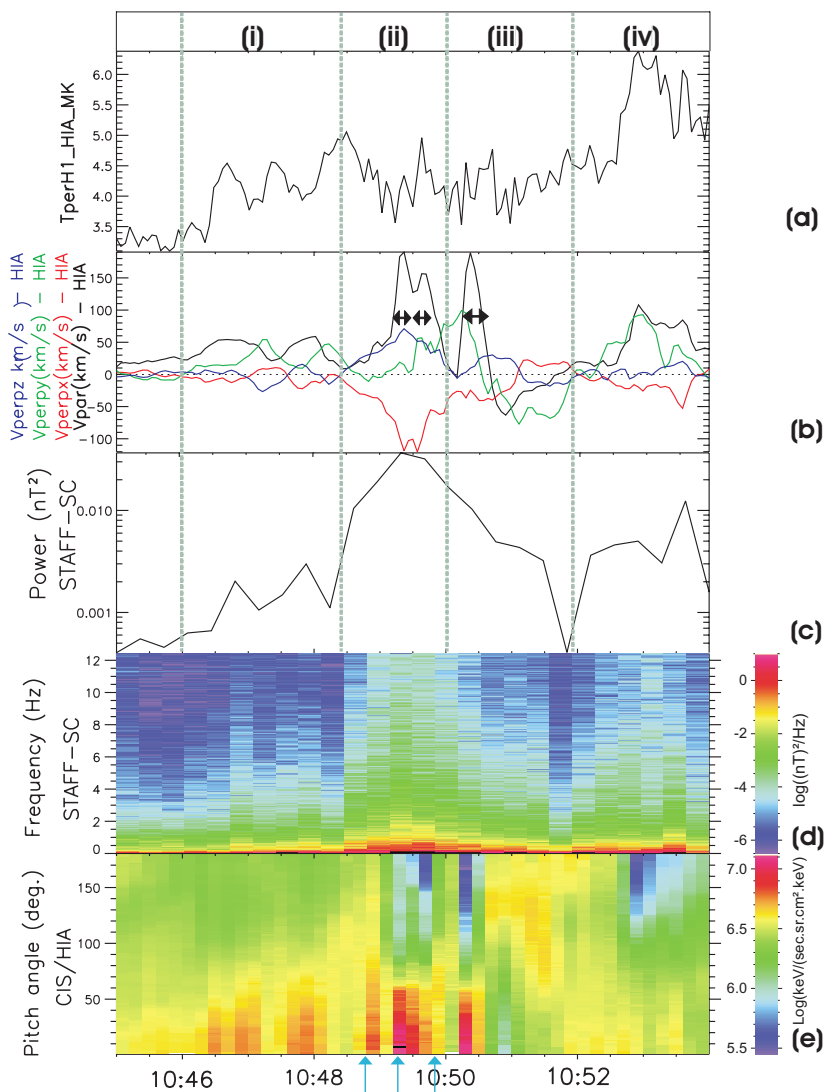

Fig. 4. This is an enlargement of an ion injection event. From top to bottom, are displayed the ion perpendicular temperature measured by HIA (a), the bulk flow velocity given by HIA projected along the magnetic field direction given by FGM (in black) and the GSM components of the perpendicular bulk flow velocity (b), the magnetic wave power integrated between 1 and $10 \mathrm{~Hz}$ from STAFF-SC (c), the corresponding time-frequency spectrogram (d) and the ion pitch angle spectrogram given by HIA (e). Four successive periods - (i) to (iv) - are delimited by dotted vertical lines. Vertical blue arrows at the bottom of the figure indicate the centre time of the distribution function shown in Fig. 5. Horizontal black arrows on panel (b) delimit the time intervals used in Sect. 4.

Later (after 11:01 UT), the absence of ion beams is probably due to the fact that the farther the spacecraft move sunward across the cusp, the farther they are from the region linked to the reconnection site in the northern lobe.

The conjunction of an injection of magnetosheath ions and an electromagnetic wave activity has often been noticed (e.g. D'Angelo et al., 1974; Le et al., 2001). From 10:53 to 11:22 UT, the large peaks of the perpendicular flow velocity are associated with small magnitudes of the parallel flow velocity. In contrast with the injection events, the plasma there does not consist of a predominant earth-going plasma but also includes counterstreaming mirrored ions. Periods where upgoing and downgoing ions are of the same intensity (near zero parallel flow velocity) correspond to the periods where the convection is maximum (large perpendicular flow velocity) and where a strong electromagnetic activity is observed. On the other hand, when mirroring ions predominate (negative parallel flow velocity), both the wave activity and the plasma convection are systematically weaker. As a result, it can be thought that some wave activity also results when a large convection is present; electromagnetic wave activity is thus not only observed during ion beams events.

At 11:20 UT, the IMF $B_{y}$ is no longer negative and the clock angle moves close to $0 \mathrm{deg}$. This IMF component is known to influence the cusp location significantly (Russell, 2000). It is thus possible that this IMF turning resulted in the entry of the spacecraft in the stagnant part of the highaltitude cusp. This region shows very low flow and magnetic field; as suggested by Lavraud et al. (2004), this may be typical for northward IMF conditions. The plasma displays neither unidirectional nor counterstreaming ion beams, but rather appears fairly isotropic. In this region, both electric and magnetic fluctuations are very low (see Fig. 3).

\subsection{Focus on an ion injection event}

Figure 4 presents focuses on the last succession of strong ion injections associated with waves, between 10:45 and 10:54 UT. From top to bottom, the ion perpendicular temperature, the ion velocity components, the integrated power of the ULF magnetic wave, the time-frequency power spectrogram of these waves, and the ion pitch angle spectrogram are displayed. The event has been divided into four successive intervals. The frontiers have been fixed where the parallel flow velocity is close to 0 . The (ii) period covers the first and second strong injections. The (iii) period includes the third strong injection and the consecutive mirrored ions. The perpendicular temperature (panel a) is low (less than 3.5 MK) before interval (i), fluctuates during intervals (i), (ii) and (iii) and is high (more than $4.5 \mathrm{MK}$ ) later. The parallel flow velocity (panel (b), black curve) displays two major positive peaks: at 10:49:20 and 10:50:20 UT, the parallel velocity reaches almost $200 \mathrm{~km} / \mathrm{s}$. Between these two peaks, it passes through a secondary maximum followed by a brief moment of vanishing velocity. After the second major peak, the parallel velocity is negative until 10:51:30 UT. Then it turns back to positive. During the (i) interval, all the components of the perpendicular bulk flow velocity display low values (less than $50 \mathrm{~km} / \mathrm{s}$ ). During the first strong injection (interval (ii)), $v_{x}$ is strongly negative and passes through an extremum of about $-100 \mathrm{~km} / \mathrm{s}$ at 10:49:30 UT. In the (iii) interval, the perpendicular velocity fluctuations occur mainly along the $y$-direction. The $v_{y}$ component is dawnward when outgoing ions dominate and is duskward elsewhere. The ULF electromagnetic wave activity starts to increase at 10:48:10 UT by a factor of 30 (panels $\mathrm{c}$ and $\mathrm{d}$ ). It is maximum around 10:49:15 UT. The level decreases until 10:51:20 UT, almost by a factor of 100 . Then after that it fluctuates around an intensity level of one-tenth of the maximum. On the pitch angle spectrogram (panel c), the two strong bursts of mainly fieldaligned ions are observed just before and after 10:50 UT, during periods (ii) and (iii). During interval (i), two other fieldaligned beams are also detected but they are less intense. It 

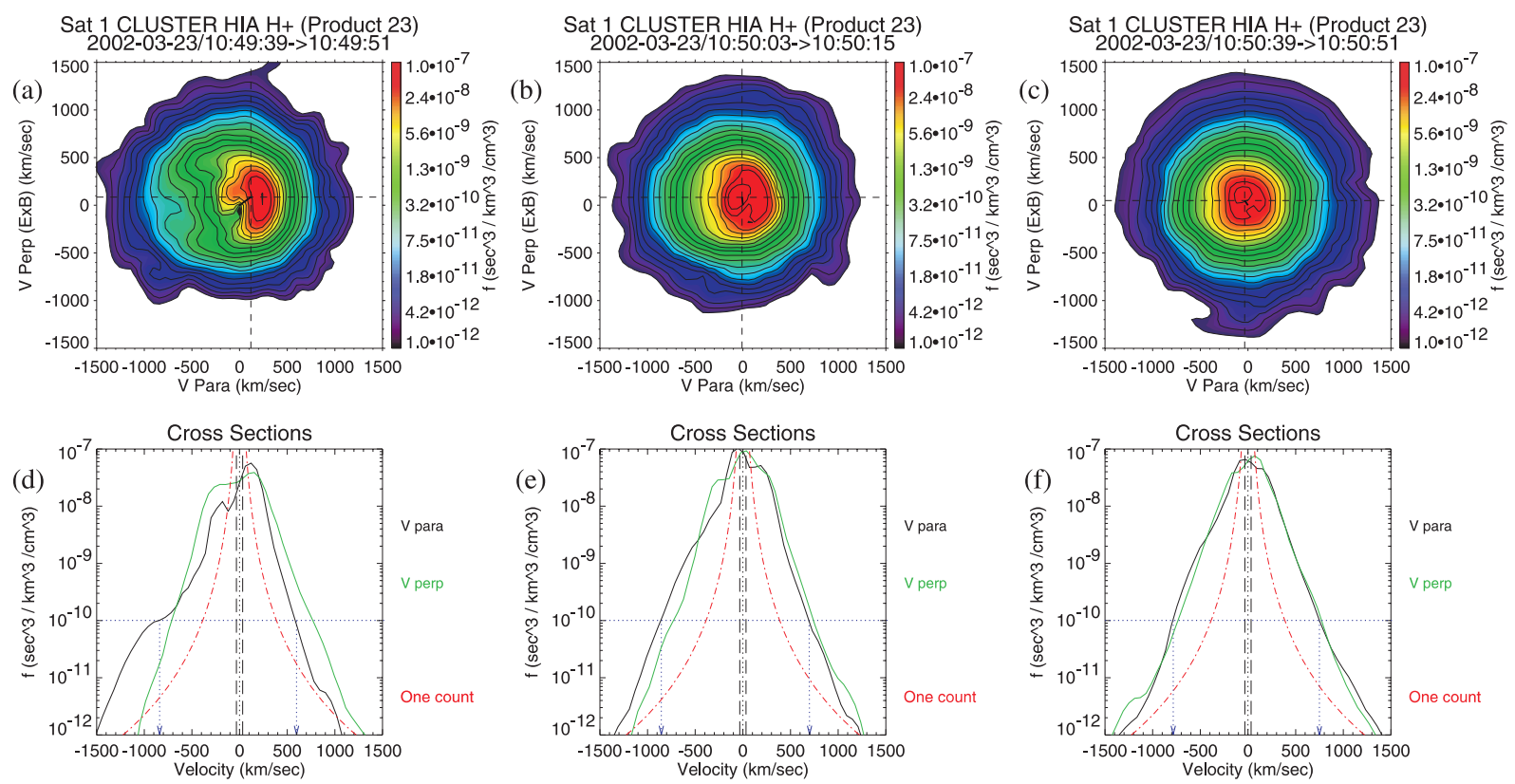

Fig. 5. Ion distribution functions of the ions from HIA on board spacecraft 1 for three selected times during the interval of interest. Twodimensional cuts of the ion distribution, in the parallel-perpendicular plane (the perpendicular direction is that of the convection), are shown in plots (a), (b) and (c). Their respective 1-D cuts along the parallel and perpendicular direction (black and green, respectively) are given in plots (d), (e) and (f). The one count level is superimposed to these latter as red dash-dotted lines. The dotted blue lines in plots (d), (e) and (f) only serve as guides to highlight the distributions' width. Distributions (a), (b) and (c) are presented in the spacecraft frame (dotted black lines indicate the flow velocities used to define the plasma frame). Distributions (d), (e) and (f) are presented in the plasma frame.

seems that they are not located on freshly reconnected field lines because of numerous upgoing ions. Later, more diffuse (but for a longer time), anti-parallel ions are detected.

Figure 5 shows 2-D and 1-D cuts of the ion distribution functions for three selected times (shown by blue arrows in Fig. 4). The maximum of the wave activity is reached when the parallel component of the flow velocity is the largest (and positive). It corresponds to an anisotropic distribution function (cuts (a) and (d) of Fig. 5). After the last injection the ions have been reflected at low altitudes and return to the spacecraft, resulting in a bulk flow with a substantial antiparallel component (part (iii) in Fig. 4). There, the wave activity turns back to a lower level. The velocity changes are sharper when the precipitating ions (parallel population) are dominant while smoother when mirrored ions are largely present. It is hard to disentangle whether this stems from ballistic effects (the presence of both up- and downgoing ions on the same field line may render the velocity changes smoother) or from wave-particle diffusion processes. In the following interval (part (iv)), a little injection is observed and the wave activity has a mean level. These observations strongly suggest a causal link between the wave generation and the injection of particles. This is also supported by the observations of local enhancements of the wave activity during the two ion beams of interval (i). It is worth noticing that the first strong injection period (between 10:49:20 and 10:49:45 UT) is associated with a strong antisunward convection motion. We remember here that the $B_{z}$ orientation of the IMF was unclear at this time (turned from northward to southward at 10:51 UT). Although the site location cannot be deduced from either the IMF orientation or from a time-latitude dispersion analysis, due to very short duration of the event, the convection motion argues for a reconnection site located in the equatorward region of the magnetopause. Note also that such a location is consistent with either southward or northward IMF (Fuselier et al., 2000). The second strong injection period (at 10:50:20 UT) has a large duskward component that is coherent with an anti-parallel merging site located more dawnward from the cusp (the $B_{y}$ component of the IMF is directed dawnward). This focus on a short interval allowed us to highlight the apparent simultaneity of wave activity and injections of magnetosheath ions. It further confirms the relationship between the ion flux intensity (when directed earthward) and the power of the ULF electromagnetic wave activity.

The large field-aligned flows (and fluxes) are characteristic of newly-open field lines; the precipitating magnetosheath ions, characterized by large parallel velocities, are detected before the mirrored component, owing to time-of-flight effect. The temporal evolution of the ion distribution functions displayed in Fig. 5 highlights this feature. The first distribution (a) was measured during the large flows and appears D-shaped, compatible with expectations from the reconnection model (e.g. Smith and Lockwood, 1996). It then evolves with the gradual detection of large fluxes at all pitch angles in distributions (b) and (c). To assess whether a 

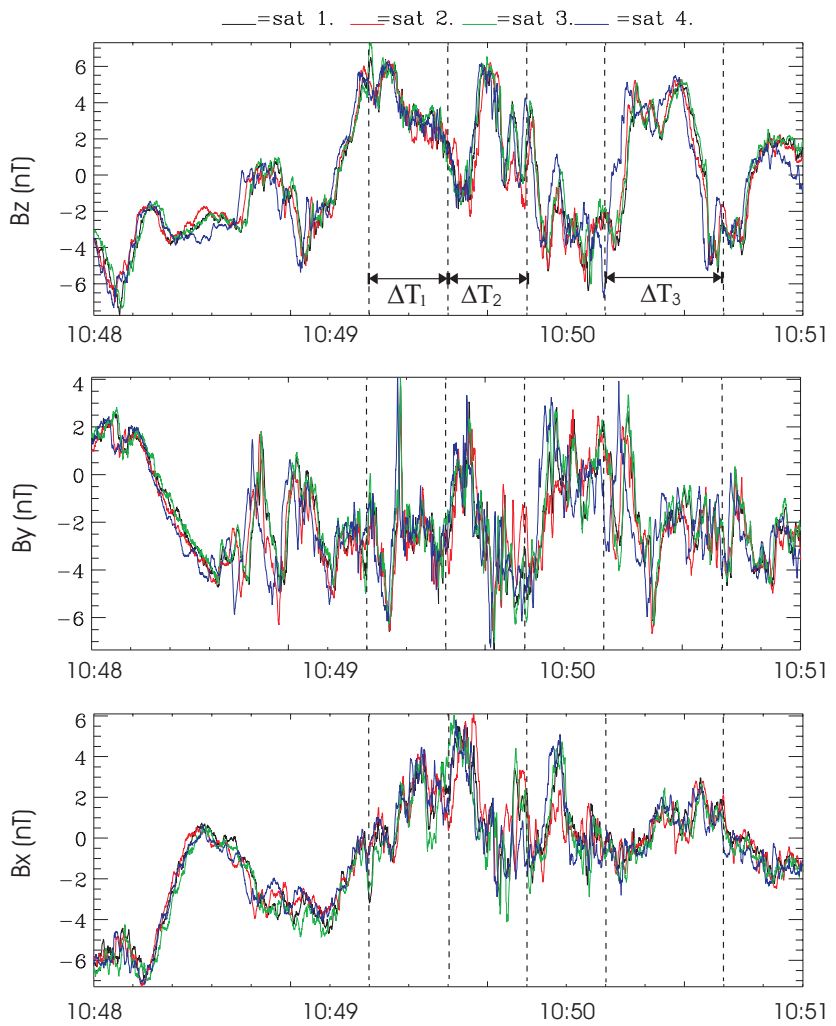

Fig. 6. The magnetic field components measured by the FGM experiment in the MFA frame over $3 \mathrm{~min}$, starting from 10:48:00 UT. The mean values (over time and spacecraft) are removed from the signal and used to define the MFA frame. The colours are relative to the four satellites. The vertical dashed lines delimit the intervals when the maxima of the parallel flow velocities are observed.

wave-particle interaction may also contribute to the evolution of the distribution, one may first compare the parallel and anti-parallel components of the distributions (d), (e) and (f). The 1-D cuts of the distributions displayed in plot (e) reveal that the width of the distribution in the anti-parallel direction is larger than that in the parallel direction. Sundkvist et al. (2005) highlighted that waves are generated in the cusp along whole field lines, meaning above and below the Cluster spacecraft. Then considering that the mirrored ions spend more time than the downgoing ones in crossing the region where electromagnetic waves can interact with the ions, heating will be more important for the mirrored particles. It is worth noticing that the next distribution (f) shows only little evidence for the same feature. On the other hand, ion heating may equally occur above the spacecraft location. This suggestion comes from the appearance of a high-energy tail in the parallel spectrum of the ions in distribution (f), as compared to that of distribution (d). Our observations may therefore represent some heating which occurs during a major part of the trajectory of the ions after their injection into the magnetosphere.

We must stress, however, that the observations of the distribution function characteristics in the parallel direction are experimentally difficult because of the mixing of the up- and downgoing populations. The presence of these two populations induces uncertainty in the determination of the plasma frame and makes any assessment from the parallel spectra - in the present case a difference in temperature - not fully conclusive. Assuming gyrotropy, one may expect the analysis of the perpendicular temperature to be less sensitive to this problem. A lack of perpendicular temperature enhancement would suggest the absence of heating, while the detection of a finite temperature enhancement would constitute an upper limit to any given heating mechanism. From the time series of the perpendicular temperature shown in Fig. 4, the lowest perpendicular temperature during the large flows is about 3.5 MK, while it is of about 4.5 MK later when the distribution is isotropic, for example, at 10:52 UT. There is an upper limit of a $30 \%$ increase in temperature for this particular case, as more and more mirrored particles are detected. This, added with the observations in the parallel temperature, suggests that some heating may occur by wave-particle interaction in the cusps. Nevertheless, it should be compared with the typical factor of 2-3 observed between the magnetosheath temperature and that of the exterior cusp (at their boundary) under northward IMF (Lavraud et al., 2004, 2002). The other processes at other locations (e.g. reconnection site or magnetopause discontinuity) are probably also needed to explain the temperatures observed in the cusp, in comparison with temperatures in the magnetosheath.

\section{Mode identification using the k-filtering technique}

In this section we propose to study the nature of the wave activity discussed in the previous section. Figure 6 presents the three components of the magnetic field measured by the FGM experiment during the strong injections observed between 10:48 and 10:51 UT. They are shown in the MFA (Magnetic Field Aligned) frame, with the $z$-axis aligned with the ambient magnetic field. The mean values, calculated over the period studied and over the four spacecraft, have been removed from the waveforms and are used to define the MFA frame. We have subdivided the time interval into three sub-intervals, centred around the injection events where a maximum of the parallel velocity of the ions is observed: $\Delta T_{1} \equiv 10: 49: 10$ to $10: 49: 30 \mathrm{UT} ; \Delta T_{2} \equiv 10: 49: 30$ to 10:49:50 UT and $\Delta T_{3} \equiv 10: 50: 10$ to 10:50:40 UT (see blue arrows at the bottom of panel (b) in Fig. 4). The three injection events seem to be well correlated with large-scale fluctuations of the parallel component of the magnetic field. However, only the two first injections are accompanied by strong large-scale fluctuations (about the duration of the interval) of the perpendicular magnetic field component, in this case $B_{x}$. Perpendicular fluctuations of smaller scales (duration of few seconds) are seen in each of these injections at 10:49:16, 10:49:35 and 10:50:08 UT on the $B_{y}$ component.

First, the analysis is performed during the period $\Delta T_{1}$. Figure 7 presents the FFT power spectra calculated for this interval. For frequencies below $f=0.13 \mathrm{~Hz}$, the 
parallel component of the magnetic field is larger than the perpendicular one. This tendency is reversed for frequencies ranging from 0.13 to $1.0 \mathrm{~Hz}$. The dominance of the perpendicular component in the frequency range $[0.13,1.0] \mathrm{Hz}$, as expected from the inspection of the waveforms, can be interpreted as a signature of shear waves. To confirm this interpretation, we have used the k-filtering technique to identify the nature of the waves for each frequency in the interval $[0.13,1.0] \mathrm{Hz}$. Before presenting the results obtained, let us recall the principle of the k-filtering method (Pinçon and Lefeuvre, 1991; Pinçon and Motschmann, 1998).

The k-filtering technique is based on the simultaneous measurements of a given wave field in several points of space. It allows one to estimate the energy distribution function of the corresponding wave field in the 4-D Fourier space $(\omega, \mathbf{k})$, denoted hereafter by the function $P(\omega, \mathbf{k})$. The kfiltering method adopts a plane wave decomposition and requires the time stationarity and space homogeneity of the time series. In real data, these two hypotheses are not strictly fulfilled. However, in practice, we are content with the concept of weak space homogeneity (and time stationarity): the signal should be homogeneous (stationary) on scales that are larger than the largest spatial (temporal) scale determined by the k-filtering method. Once the wave energy distribution $P(\omega, \mathbf{k})$ is calculated, it can be used to identify the relevant modes (Sahraoui et al., 2003). For each given frequency $\omega_{0}$, and using an isocontour representation, the energy distribution in the $\mathbf{k}$-space is displayed as cuts in the $\left(k_{x}, k_{y}\right)$ plane along the $k_{z}$ axis. The validity domain of the technique in the wave vector space is determined from the separations between the Cluster spacecraft: all the existing wavelengths have to be larger than the intercraft separations, which are of the order of $100 \mathrm{~km}$ in the present case. For each value of $k_{z}$ corresponding to an identified peak of $P\left(\omega_{0}, \mathbf{k}\right)$, the theoretical dispersion relations of the low-frequency (LF) linear plasma modes (MHD and mirror modes) are superimposed on the energy spectrum (after being Doppler shifted into the spacecraft frame using the flow velocity measured by the HIA experiment (Rème et al., 2001)). The mirror mode is assumed to have approximately a zero frequency in the plasma frame, which means that it is observed in the satellite frame with the dispersion $\omega=$ k.v. When it is necessary, other theoretical dispersion relations (kinetic modes), computed from the WHAMP program (Rönnmark, 1982), are introduced besides the MHD modes. For both MHD and kinetic modes the control parameters are given by the different Cluster experiments: $\mathbf{B}_{0}$ from FGM, the ion temperature and density from HIA. For more details on the use of the k-filtering technique, the reader is referred to Sahraoui et al. (2003) and the references therein.

In the following we present the results obtained in the frequency range $[0.13,1.0] \mathrm{Hz}$. The frequencies below $0.35 \mathrm{~Hz}$ are studied from the FGM data, whereas the higher frequencies are studied from the STAFF-SC data. In Sahraoui et al. (2004), it has been shown, from the very good similarity between STAFF-SC and FGM results, that there is no effect of the high-pass filter applied to STAFF-SC data

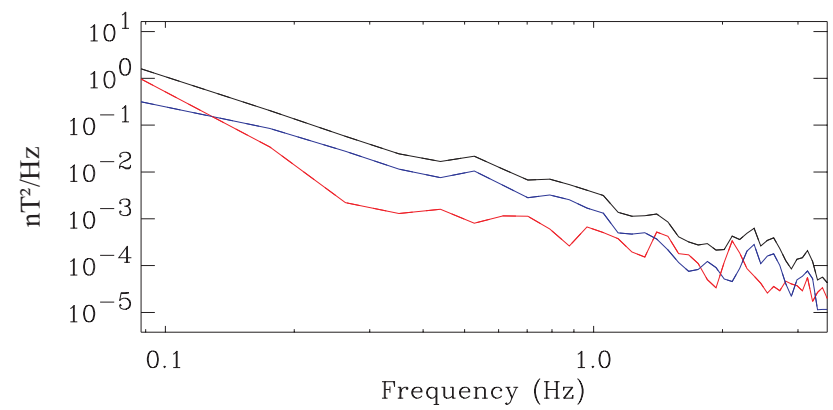

Fig. 7. The FFT power spectra of the FGM data during the period $\Delta T_{1}$ calculated for the spacecraft 1 in the frequency range $[0.09,3] \mathrm{Hz}$. The red and blue lines show the spectra of the parallel and perpendicular components, respectively. The black line shows the total spectrum.

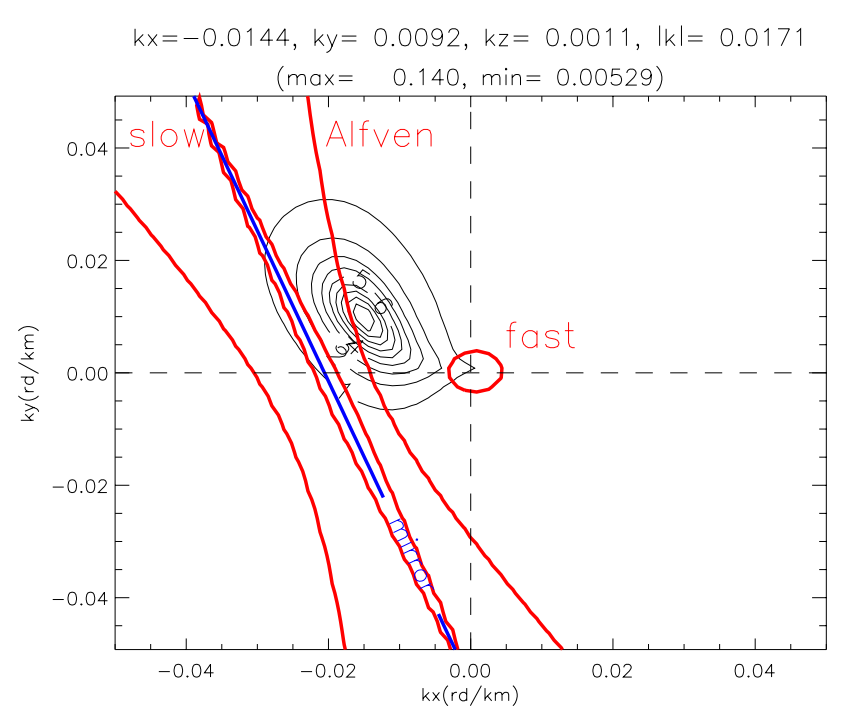

Fig. 8. Energy distribution in the $\mathbf{k}$-space of the most intense identified peak for the frequency $f=0.26 \mathrm{~Hz}$. It is presented in the $k_{z}=0.0011 \mathrm{rd} / \mathrm{km}$ plane which contains the maximum of magnetic energy for this frequency. The black thin lines are the isocontours of energy in the $\left(k_{x}, k_{y}\right)$ plane, whereas the coloured lines are the theoretical dispersion relations of the LF modes Doppler shifted in the satellite frame. The blue line is the Doppler shift $\omega=\mathbf{k . v}$ and corresponds to the mirror dispersion relation in the satellite frame. The curves of the slow mode are very close to the curve of the mirror mode, since the slow mode propagates in the present case quasiperpendicularly to the magnetic field.

on the spatio-temporal properties of the observed waves. For the frequency $f=0.26 \mathrm{~Hz}$, the $\left(k_{x}, k_{y}\right)$-plane, containing the maximum of the magnetic energy distribution function, is shown in Fig. 8. It is found for $k_{z}=0.0011 \mathrm{rd} / \mathrm{km}$ which is the smallest wave number resolved with the k-filtering method. As one can see, the energy maximum lies very close to the theoretical dispersion relation of the Alfvén mode. The observed mode has a wave number quasi-perpendicular to the magnetic field $\left(\theta=\left(\mathbf{k}, \mathbf{B}_{0}\right) \sim 86^{\circ}\right)$. 

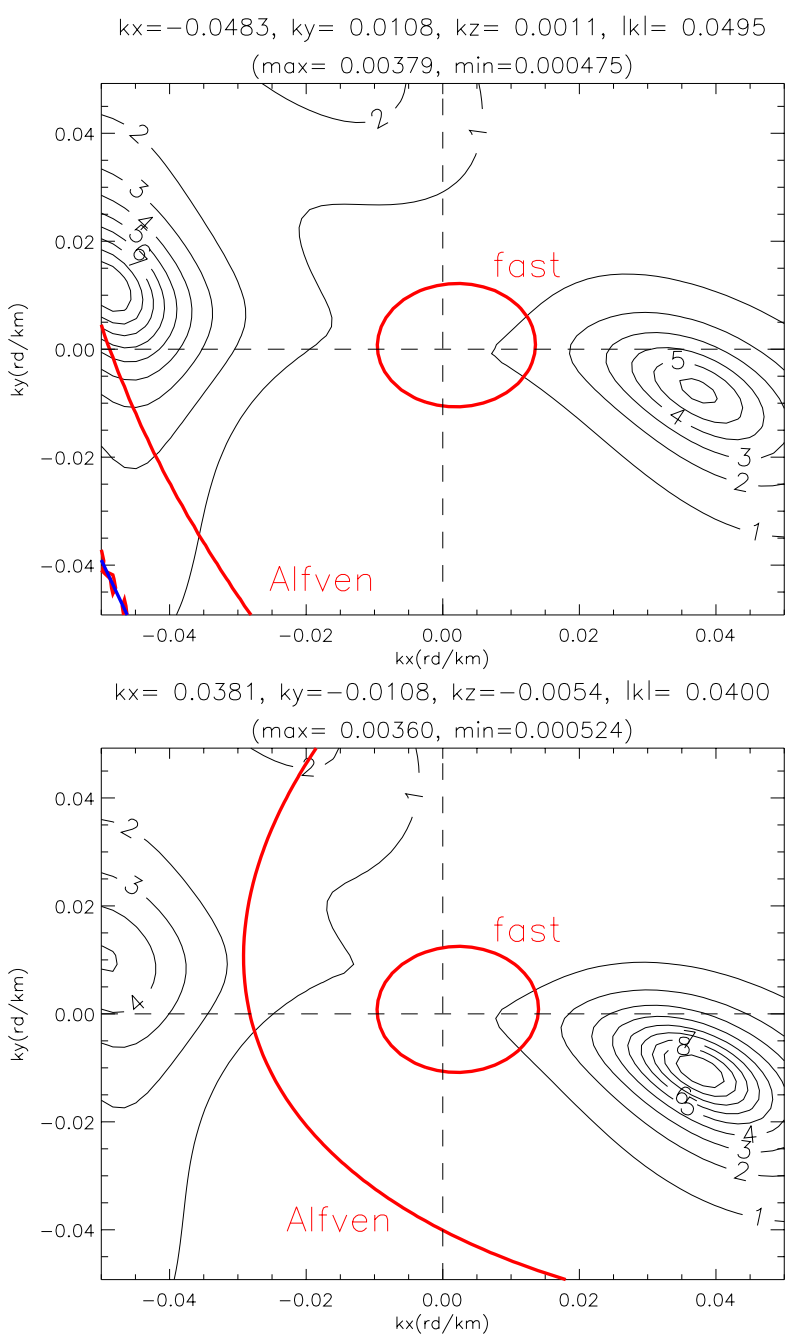

Fig. 9. Two peaks with comparable intensities are identified for the frequency $f=0.79 \mathrm{~Hz}$. The first one (left-hand side of the top panel) corresponds to the latest identifiable Alfvén mode with $\left(\mathbf{k}, \mathbf{B}_{0}\right)=88^{\circ}$ and $f_{\text {plasma }} \approx 0.5 f_{c i}$. The second one (right-hand side of the bottom panel) is identified by WHAMP as a Bernstein mode with: $\left(\mathbf{k}, \mathbf{B}_{0}\right)=98^{\circ}$ and $f_{\text {plasma }} \approx 2.5 f_{c i}$.

Now, one may ask whether the identification of the Alfvén mode for this frequency is consistent for higher frequencies. For this purpose, we have analyzed all the frequencies of the interval $[0.1,1.0] \mathrm{Hz}$. Figure 9 presents the $\left(k_{x}, k_{y}\right)$ planes containing the two main energy peaks for the frequency $f=0.79 \mathrm{~Hz}$. At this frequency, the first and second extrema of energy lie, respectively, in planes for which $k_{z}=0.0011 \mathrm{rd} / \mathrm{km}$ (top) and $k_{z}=-0.0054 \mathrm{rd} / \mathrm{km}$ (bottom). The maximum energy is on the left edge of the top panel (i.e. $k_{x} \approx-0.05 \mathrm{rd} / \mathrm{km}$ ) and it is close from the theoretical Alfvén mode. In this panel, significant energy is also present around the point of coordinates $k_{x} \approx 0.037 \mathrm{rd} / \mathrm{km}$ and $k_{y} \approx-0.008 \mathrm{rd} / \mathrm{km}$. It is worth noticing that these coordinates correspond to the location of the second maximum (bottom panel): the wave energy distribution spreads over a wide range of $\mathbf{k}$. For the same reason, one can notice a peak energy on the left side of the bottom panel at the location of the maximum peak of the top panel. The two main energy peaks are found for a parallel wave number which is small as compared to the perpendicular one. The study confirms the observation of the Alfvén mode up to the frequency $f=0.8 \mathrm{~Hz}$, where it reaches the shortest wavelength accessible to the measurement given the 100-km intercraft separation (see Fig. 9, in the left-hand side of the top panel, the maximum is on the edge of the box). As the frequencies increase, the wave vectors of the identified Alfvén mode have constant parallel components (all corresponding peaks are in the same $k_{z}$ plane) but increasing perpendicular ones, leading to various propagation angles (with respect to the local ambient magnetic field), ranging from $83^{\circ}$ to $88^{\circ}$. The modulus of the wave vectors is varying from $k \rho=0.8$ for $f=0.18 \mathrm{~Hz}$ to $k \rho=3.7$ for $f=0.8 \mathrm{~Hz}$ (as seen in Fig. 10, described below), where $\rho \sim 75 \mathrm{~km}$ is the proton Larmor radius. We notice therefore that the appellation "Alfvén mode" used in the present work should be taken with some caution: the identified spatial scales are typically in the range of the kinetic regime of the Alfvén mode and the name "Kinetic Alfvén Wave" would be a more accurate name of the Alfvénic fluctuations observed in the present case. Nevertheless, for the sake of simplicity, we remain in the classical "Alfvén mode" appellation most of the time. In line with this, we notice that for such spatial scales $(k \rho \sim 3)$ the properties of the Alfvén mode are much different from those corresponding to the MHD limit $(k \rho \ll 1)$. The Alfvén mode, even in the kinetic range, is expected to have a group velocity mainly directed along the field line. For instance, the compressibility of the mode, defined as the ratio $\delta B_{/ /} / \delta B_{\perp}$ and calculated from WHAMP, varies from zero in the MHD limit to 0.4 for $k \rho \sim 3$. The identification of the Alfvén mode as the dominant one in the frequency range $[0.1,0.8] \mathrm{Hz}$ over a wide range of spatial scales allows one therefore to explain the dominance of the perpendicular magnetic component with respect to the parallel one mentioned above, but also some of the observed parallel fluctuations. Other compressional modes with lower intensity (not shown here) are identified in the same range of frequency. Their detailed study is postponed to future works.

For the frequency $f=0.8 \mathrm{~Hz}$, in addition to the Alfvén mode peak, we have identified another peak with a comparable energy. It is localized in the $k_{z}=-0.0054 \mathrm{rd} / \mathrm{km}$ plane (Fig. 9, right-hand side of the bottom panel). Its extension to other $k_{z}$ planes appears on the top panel of Fig. 9. This wave has approximately the same spatial scales as the observed kinetic Alfvén wave: both wave vectors are almost equal but have opposite sign, which means that they have been symmetrically Doppler shifted, according to the formula $\omega_{\text {plasma }}=\omega_{\text {satellite }} \pm$ k.v. However, from Fig. 9 (righthand side of the bottom panel), we see that this second peak is located far from any dispersion curve of the MHD modes. The corresponding frequency in the plasma frame is approximately $f=1.3 \mathrm{~Hz} \sim 2.5 f_{c i}$, where $f_{c i}$ is the proton gyrofrequency.

Bernstein modes are known to have dispersion relations in kinetic range close to $\omega=(n+0.5) . \omega_{c i}(n>1)$. Using WHAMP, we have identified this wave as an $n=2$ Bernstein 
mode. The Bernstein modes are very unstable for the quasi-perpendicular direction, and are generally electrostatic (Janhunen et al., 2003). However, in this case of large wave vectors $(k \rho \sim 3.5)$ and large $\beta(\approx 1)$, WHAMP provides an important magnetic component for the Bernstein mode: $\delta E / \delta B \sim 4 V_{A}$, where $V_{A} \approx 350 \mathrm{~km} / \mathrm{s}$ is the Alfvén velocity. This explains why the Bernstein mode could be present in magnetic data. This Bernstein mode is identifiable for all the frequencies belonging to $[0.8,1.0] \mathrm{Hz}$, and disappears for higher ones. Its wave frequency in the plasma frame decreases with the wave vector. The observed wave vectors form angles varying from $99^{\circ}$ to $95^{\circ}$ with respect to $\mathbf{B}_{0}$.

In order to summarize all the previous results, we present in Fig. 10 the dispersion relations observed for the two principal identified wave peaks. The frequencies measured in the spacecraft frame are Doppler-shifted back into the plasma frame and then plotted as a function of its perpendicular wave vector (i.e. $\omega_{\text {plasma }}$ versus $k_{\perp}$ ), the dependence with the parallel wave vector being negligible. These experimental dispersion relations are compared to the theoretical ones obtained from WHAMP. The question of de-Dopplerizing has to be treated with caution, since strong variations of the plasma velocities are observed during the period studied, $\Delta T_{1}$ (see Fig. 4). The crucial point is to disentangle the velocity perturbations caused by the waves from the background flow velocity. For this purpose three different values of the plasma velocity have been used to Doppler-shift the wave frequencies measured in the satellite frame back into the plasma frame. These values are calculated in the MFA frame as follows:

- $\mathbf{v}_{1}=(-100,-40,140) \mathrm{km} / \mathrm{s}$, as averaged over $\Delta T_{1}$ (20s),

- $\mathbf{v}_{2}=(-50,-16,58) \mathrm{km} / \mathrm{s}$, as averaged over $180 \mathrm{~s}$ between 10:48:00 and 10:51:00,

$-\mathbf{v}_{3}=\left(\mathbf{v}_{1}+\mathbf{v}_{2}\right) / 2=(-75,-28,-100) \mathrm{km} / \mathrm{s}$.

The velocities $\mathbf{v}_{1}, \mathbf{v}_{2}$ and $\mathbf{v}_{3}$ are used, respectively, to plot the green, blue and red experimental dispersion curves displayed in Fig. 10. Owing to the fact that the perpendicular gradients are much sharper than the parallel ones $\left(k_{/ /} \ll k_{\perp}\right)$, the Doppler-shift is less sensitive to the parallel flow velocity. It has to be stressed that even with an important uncertainty in the determination of the background flow velocity, the mode identification of the observed wave peaks is still accurate. As a matter of fact, Fig. 10 shows that the global behaviour of the experimental dispersion curves follows the ones of the Alfvén and Bernstein modes, even if they are not very close (green and blue dots). The fast mode lies far from all the experimental peaks. The slow mode (not plotted in Fig. 10) has almost a zero frequency and moreover, is strongly damped above $k \rho=1$.

We also notice that the two velocities $\mathbf{v}_{1}$ and $\mathbf{v}_{2}$ can be considered as over- and underestimations of the background flow velocity, respectively. In fact, since the wave activity presents a large-scale fluctuation over the period $\Delta T_{1}$, the mean value of the flow velocity over this period, calculated

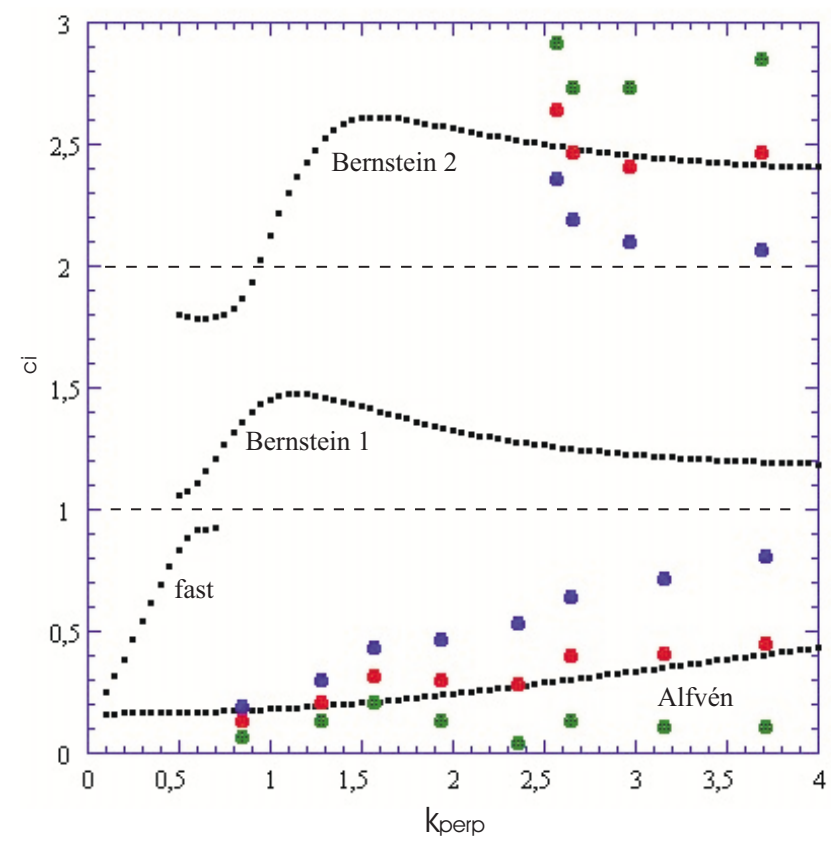

Fig. 10. Comparison of the experimental dispersion relations (coloured dots) observed in the frequency range $[0.1,1] \mathrm{Hz}$ to the theoretical ones determined from WHAMP (black curves). The blue, green and red dots are the de-Dopplerized experimental dispersions obtained with the flow velocities $\mathbf{v}_{1}, \mathbf{v}_{2}$ and $\mathbf{v}_{3}$, respectively. The dispersion of the waves observed in the frequency range $[0.1,0.8] \mathrm{Hz}$ fits the Alfvén wave dispersion curve shape, and quite well for $\mathbf{v}_{3}$ (red dots). The dispersion of the waves observed in the frequency range $[0.8,1] \mathrm{Hz}$ is very close to the dispersion curve of second harmonic of the Bernstein mode for the same $\mathbf{v}_{3}$ flow velocity (red dots).

only over the high bulk velocities of the injection, turns out to be an overestimate of the flow velocity carrying waves. The inverse situation can occur when the mean velocity is calculated over a time interval which is too large. These considerations suggest that the most realistic flow velocity could have a value ranging between the two previous limits. This hypothesis is indeed strongly supported by Fig. 10: the best fit of the experimental dispersion curves with the theoretical ones is obtained when the flow velocity $\mathbf{v}_{3}$ is used for the deDopplerization (red dots). Note that Figs. 8 and 9 have been obtained with the $\mathbf{v}_{3}$ flow velocity.

The same analysis has been performed on the two other intervals, $\Delta T_{2}$ and $\Delta T_{3}$, using the same method to Dopplershift the data back into the plasma frame. The results obtained show that the Alfvén mode also dominates during the second injection (the period $\Delta T_{2}$ ). The wave characteristics associated with the third injection display a different feature. The dominant mode, even if it lies in the k-space, also along a quasi-perpendicular direction, exhibits no frequency dispersion, with all the frequencies being very low in the plasma frame $(\approx 0.1 \mathrm{~Hz})$. Hence, the dominant mode in the last time interval corresponds to a stationary structure rather than to an Alfvén wave. This observation is discussed below. 


\section{Discussion}

The Cluster case study reported here shows that in the highaltitude polar cusp, the numerous injection events of magnetosheath ions from a reconnection site located poleward of the cusp are always observed with an intense wave activity. Using the k-filtering analysis, part of these intense waves observed simultaneously are identified as kinetic Alfvén waves. The local wave generation of kinetic Alfvén waves is still not well understood but we can argue here that a plausible mechanism may be based on a current instability (Forslund et al., 1979). As seen in Fig. 6, large-scale fluctuations $(>15 s)$ of the $B_{x}$ component give evidence for the signature of parallel currents during the two first intervals, $\Delta T_{1}$ and $\Delta T_{2}$, while no signature of a large-scale parallel current is observed during the last interval, $\Delta T_{3}$. The waves identified during the two first injections $\left(\Delta T_{1}\right.$ and $\left.\Delta T_{2}\right)$ are mostly of an Alfvénic nature and show similar characteristics. On the other hand, the third injection, which corresponds to a lower intensity in the parallel current, shows no evidence for Alfvén waves. Parallel currents may thus be the energy source of the kinetic Alfvén waves which have been observed. Nevertheless, we notice that background parameters may influence the onset of electromagnetic wave activity: specific beams could either generate or not generate kinetic Alfvén waves under various conditions. Numerical simulations are needed to clarify this issue.

In the MHD theory homework, the Alfvén mode is expected to induce a perturbation of the bulk plasma velocity of about $\delta v=v_{A} \delta B / B_{0}$. Both perturbations $\delta v$ and $\delta B$ are perpendicular to the $\left(\mathbf{B}_{\mathbf{0}}, \mathbf{k}\right)$ plane. If one considers the perturbation seen on $B_{x}$ during the two injections observed between 10:49:20 and 10:49:40 (see Fig. 10) as the temporal signature of an Alfvénic current, a perturbation on the plasma motion of about $100 \mathrm{~km} / \mathrm{s}$ along the $x$-axis is expected. It is precisely the amplitude of the perpendicular bulk flow velocity measured along the $x$-axis during that period (see Fig. 4). So, its interpretation from a large-scale Alfvén wave perturbation is consistent. Conversely, the observation of such an enhanced perpendicular plasma motion during an injection event could be the signature of a large-scale Alfvén wave.

The theory of reconnection predicts the production of Alfvén waves along newly-opened field lines (e.g. Haerendel et al., 1978). The Alfvén waves observed by Cluster may thus originate from the reconnection sites associated with the injection events. However, the close simultaneity of the wave and particle events can hardly be explained other than by a local generation of the waves. In the framework of reconnection (Smith and Lockwood, 1996), the magnetosheath ions penetrating into the magnetosphere are accelerated up to the local Alfvén velocity and move then in a first approximation with a constant velocity. The Alfvén waves generated simultaneously may therefore follow the injected ions as long as the variation of the Alfvén velocity along the field line is negligible. Indeed, if one considers an Alfvén velocity of about $200 \mathrm{~km} / \mathrm{s}$ at the reconnection site, which corresponds to the parallel flow velocity observed for the injected ions, an Alfvén velocity of about $350 \mathrm{~km} / \mathrm{s}$ at the location of the Cluster spacecraft, and a distance of about $10 R_{E}$ between the reconnection site and the spacecraft, a time lag of about $80 \mathrm{~s}$ between the detection of the magnetosheath ions and the Alfvénic perturbations may be expected in the event presented in this study (the two successive events around 10:50 UT). In fact, with the time resolution of the particle instrument (up to $4 \mathrm{~s}$ ) being much higher than $80 \mathrm{~s}$, the observations do not give any evidence for such a time lag. So, in all likelihood, the observed Alfvén wave is locally generated. Otherwise, one should describe a mechanism explaining a common propagation of the injected ions and the Alfvén wave from the reconnection site. The distance of $10 R_{E}$ has been estimated following the method presented in Onsager and Fuselier (1994). From Fig. 1, we notice that this distance is consistent with a subsolar position of the reconnection site; Vontrat-Reberac et al. (2003) obtained a similar estimation in the case of lobe reconnection. The antisunward convection motion is still the only indicator of the site location.

Concerning the waves identified as Bernstein mode the situation can be understood in a relatively simple way. As kinetic modes, the Bernstein modes are known to be very sensitive to the shape of the distribution function, which may explain why the mode identification cannot be perfectly clear. Ion distribution due to newly-reconnected field lines generates predominantly perpendicular waves (Bingham et al., 1999). More recently, Janhunen et al. (2003) defined that part of the waves destabilized from ion shell are Bernstein modes. In all likelihood, part of the waves generated from the anisotropic distribution function of the ion beam (roughly half-ring) are probably Bernstein waves. Thus, the observation of Bernstein modes during injection events is not very surprising, even if the predominance of the second harmonic over the first one is not obvious. Bernstein waves are known to hardly propagate, except perpendicularly to the magnetic field (Ichimaru, 1973). The Bernstein waves are thus very likely observed close to their source. The wave energy observed during the injection events, as dominated by the kinetic Alfvén and Bernstein modes, is thus of local origin.

The evolution of the distribution function of the magnetosheath ions that penetrate into the polar cusp on a newlyopened flux tube (in other words, during an injection event), can to a great extent be explained from the time-of-flight effect on the ions (e.g. Smith and Lockwood, 1996). As the wave intensity is linked with the beam shape of the ion distribution function, its decrease observed during the injections can also be explained from spatial considerations: the farther the spacecraft are from the ion beams (major source of the wave instabilities), the weaker the observed wave intensity. However, the role of the waves in the isotropization of the ions, even weak, cannot be ruled out. We have highlighted that the distribution of the mirrored particles (antiparallel velocities) is slightly hotter than the distribution of the downgoing ones (parallel velocities). We have also noticed an increase in the perpendicular temperature as the distribution functions become more and more isotropic. This 
suggests that some ion heating occurs along the whole field line above and below the spacecraft. Diffusion by the electromagnetic waves simultaneously detected is a good candidate to explain the local part of this heating, especially the perpendicular one. Following a classical scheme it can contribute to increase the parallel temperature (André et al., 1990).

\section{Summary and conclusions}

The main result of this study is the identification of mixed modes in the ULF turbulent-like electromagnetic spectrum observed in the high-altitude cusp region. These results are provided by the unique possibility offered by the Cluster mission, which allows the simultaneous measurements in several points of space. The presence of kinetic Alfvén waves, Bernstein waves and other less energetic waves suggests that various processes of wave generation occur. The injected proton events that have been observed are clearly associated with a strong ULF electromagnetic activity. This is a strong argument for the local generation of the waves. Many processes for the generation of kinetic Alfvén waves exist, the current instability being a plausible mechanism consistent with the observation of large-scale magnetic field fluctuations. Generation of Bernstein waves can be explained by the specific distributions of the injected ions. The associated waves (kinetic Alfvén and Bernstein waves) are good candidates to explain part of the observed ion heating. Therefore, we suggest that the electromagnetic waves contribute to the evolution of the ion distribution function associated with the injections, even if this evolution can be explained by ballistic effect. Very isotropic distribution functions, observed in the stagnant plasma region and between strong injections, correspond systematically, as well as unidirectional flows of mirrored ions, to very low wave activity. On the other hand, intense electromagnetic waves are present during periods of high plasma convection. The present case study suggests that the large-scale Alfvénic fluctuation seen in the magnetic waveform at 10:49 UT during the injection event is a signature of the strong anti-sunward convection which could be explained by the motion of the newly-reconnected flux tubes from the reconnection site equatorward of the cusp.

Finally, we stress that the scenario of generation of the Alfvén (or the Bernstein) waves, presented above, shall be taken as a first interpretation rather than as a final explanation of the observations. The study of a large variety of other cusp crossings and some theoretical developments are required to confirm these first ideas and to go further. Nevertheless, it can already be concluded that the high-altitude polar cusp, supplied with ions by the magnetosheath, acts as a source of waves, especially when the proton flows are fieldaligned. In turn, these waves, in particular, kinetic Alfvén waves and Bernstein waves, can diffuse and heat the ions along the entire trajectory of the injected ions: the process of wave generation, as observed here, is likely to occur at much lower altitudes. This hypothesis is supported here by the observation of traces of ion heating and elsewhere by previous studies (D'Angelo et al., 1974; Le et al., 2001) that showed a correlation between the waves and the injected particles at lower altitudes, also suggesting a local wave generation.

Acknowledgements. We acknowledge the ACE MAG team for the use of IMF data. STAFF data processing is partly supported by CNES grant. F. Sahraoui is funded by a CNES fellowship. The authors thank T. D. Phan for useful discussions.

Topical Editor T. Pulkkinen thanks two referees for their help in evaluating this paper.

\section{References}

André, M., Norqvist, P., Andersson, L., Eliasson, L., Eriksson, A. I., Blomberg, L., Erlandson, R. E., and Waldemark, J.: Ion energization mechanisms at $1700 \mathrm{~km}$ in the auroral region, J. Geophys. Res., 103, 4199-4222, 1998.

André, M., Temerin, M., and Gorney, D.: Resonant generation of ion waves on auroral field lines by positive slopes in ion velocity space, J. Geophys. Res., 91, 3145-3151, 1986.

André, M., Crew, G. B., Peterson, W. K., Persoon, A. M., and Pollock, C. J.: Ion heating by broadband low-frequency waves in the cusp/cleft, J. Geophys. Res., 95, 20 809-20 823, 1990.

André, M. , Behlke, R., Wahlund, J.-E., Vaivads, A., Eriksson, A.-I., Tjulin, A., Carozzi, T. D., Cully, C., Gustafsson, G., Sundkvist, D., Khotyaintsev, Y., Cornilleau-Wehrlin, N., Rezeau, L., Maksimovic, M., Lucek, E., Balogh, A., Dunlop, M., Lindqvist, P.-A., Mozer, F., Pedersen, A., and Fazakerley, A.: Multi-spacecraft observations of broadband waves near the lower hybrid frequency at the Earthward edge of the magnetopause, Ann. Geophys., 19, 1471-1481, 2001,

SRef-ID: 1432-0576/ag/2001-19-1471.

Balogh, A., Carr, C. M., Acũna, M. H., Dunlop, M. W., Beek, T. J., Brown, P., Fornaçon, K.-H., Georgescu, E., Glassmeier, K. H., Harris, J., Musmann, G., Oddy, T., and Schwingenschuh, K.: The Cluster Magnetic Field Investigation: overview of in-flight performance and initial results, Ann. Geophys., 19, 1207-1217, 2001 ,

SRef-ID: 1432-0576/ag/2001-19-1207.

Bingham, R., Kellett, B. J., Cairns, R. A., Dendy, R. O., and Shukla, P. K.: Wave generation by ion horseshoe distributions on auroral field lines, Geophys. Res. Lett., 26, 2713-2716, 1999.

Bouhram, M., Malingre, M., Jasperse, J. R., Dubouloz, N., and Sauvaud, J.-A.: Modeling transverse heating and outflow of ionospheric ions from the dayside cusp/cleft, 2 Applications, Ann. Geophys., 21, 1773-1791, 2003,

\section{SRef-ID: 1432-0576/ag/2003-21-1773.}

Cattell, C., Johnson, L., Bergmann, R., Klumpar, D., Carlson, C., McFadden, J., Strangeway, R., Ergun, R., Sigsbee, K., and Pfaff, R.: FAST observations of discrete electrostatic waves in association with down-going ion beams in the auroral zone, J. Geophys. Res., 12-1, 2002.

Chaston, C. C., Bonnell, J. W., Carlson, C. W., McFadden, J. P., Ergun, R. E., Strangeway, R. J., and Lund, E. J.: Auroral ion acceleration in dispersive Alfvén waves, J. Geophys. Res., 109, A04205, 2004.

Cornilleau-Wehrlin, N., Chanteur, G., Perraut, S., Rezeau, L., Robert, P., Roux, A., de Villedary, C., Canu, P., Maksimovic, M., de Conchy, Y., Lacombe, D. H. C., Lefeuvre, F., Parrot, M., 
Pinçon, J. L., Décréau, P. M. E., Harvey, C. C., Louarn, P., Santolik, O., Alleyne, H. S. C., Roth, M., Chust, T., Le Contel, O., and Staff Team: First results obtained by the Cluster STAFF experiment, Ann. Geophys., 21, 437-456, 2003,

SRef-ID: 1432-0576/ag/2003-21-437.

Cowley, S. W. H.: The causes of convection in the earth's magnetosphere - A review of developments during the IMS, Rev. Geophys. Space Phys., 20, 531-565, 1982.

D'Angelo, N., Bahnsen, A., and Rosenbauer, H.: Wave and Particle Measurements at the Polar Cusp, J. Geophys. Res., 79, 31293134, 1974.

Dungey, J. W.: Interplanetary Magnetic Field and the Auroral Zones, Phys. Rev. Lett., 6, 47-48, 1961.

Dungey, J. W.: The structure of the exosphere or adventures in velocity space, in Geophysics: The Earth's Environment, 526536, (Eds.) Dewitt, C., Hieblot, J., and Lebeau, A., Gordon and Breach, Newark, N. J., 1963.

Farrugia, C., Lund, E., Sandholt, P., Wild, J., Cowley, S., Balogh, A., Mouikis, C., Möbius, E., Dunlop, M., Bosqued, J., Carlson, C., Parks, G., Cerisier, J., Kelly, J., Sauvaud, J., and Rème, H.: Pulsed flows at the high-altitude cusp poleward boundary, and associated ionospheric convection and particle signatures, during a Cluster - FAST - SuperDARN- Søndrestrøm conjunction under a southwest IMF, Ann. Geophys., 22, 2891-2905, 2004,

\section{SRef-ID: 1432-0576/ag/2004-22-2891.}

Forslund, D. W., Kindel, J. M., and Stroscio, M. A.: Current driven electromagnetic ion cyclotron instability, Journal of Plasma Physics, 21, 127-139, 1979.

Fuselier, S. A., Trattner, K. J., and Petrinec, S. M.: Cusp observations of high- and low-latitude reconnection for northward interplanetary magnetic field, J. Geophys. Res., 105, 253-266, 2000.

Gurnett, D. A. and Frank, L. A.: Plasma waves in the polar cusp Observations from Hawkeye 1, J. Geophys. Res., 83, 1447-1462, 1978.

Gustafsson, G. , André, M., Carozzi, T., Eriksson, A. I., Fälthammar, C.-G., Grard, R., Holmgren, G., Holtet, J. A., Ivchenko, N., Karlsson, T., Khotyaintsev, Y., Klimov, S., Laakso, H., Lindqvist, P.-A., Lybekk, B., Marklund, G., Mozer, F., Mursula, K., Pedersen, A., Popielawska, B., Savin, S., Stasiewicz, K., Tanskanen, P., Vaivads, A., and Wahlund, J-E.: First results of electric field and density observations by Cluster EFW based on initial months of operation, Ann. Geophys., 19, 1219-1240, 2001 ,

\section{SRef-ID: 1432-0576/ag/2001-19-1219.}

Haerendel, G., Paschmann, G., Sckopke, N., and Rosenbauer, H.: The frontside boundary layer of the magnetosphere and the problem of reconnection, J. Geophys. Res., 83, 3195-3216, 1978.

Ichimaru, S.: Basic principles of plasma physics: a statistical approach, Benjamin/Cummings, Reading, Massachusets, 1973.

Janhunen, P., Olsson, A., Vaivads, A., and Peterson, W. K.: Generation of Bernstein waves by ion shell distributions in the auroral region, Ann. Geophys., 21, 881-891, 2003,

SRef-ID: 1432-0576/ag/2003-21-881.

Kintner, P. M., Labelle, J., Scales, W., Yau, A. W., and Whalen, B. A.: Observations of plasma waves within regions of perpendicular ion acceleration, Geophys. Res. Lett., 13, 1113-1116, 1986.

Lakhina, G. S.: Generation of ULF waves in the polar cusp region by velocity shear-driven kinetic Alfvén modes, Astrophys. Space Sci., 165, 153-161, 1990.

Lavraud, B., Dunlop, M. W., Phan, T. D., Rème, H., Bosqued, J.-M., Dandouras, I., Sauvaud, J.-A., Lundin, R., Taylor, M. G. G. T.,
Cargill, P. J., Mazelle, C., Escoubet, C. P., Carlson, C. W., McFadden, J. P., Parks, G. K., Moebius, E., Kistler, L. M., Bavassano-Cattaneo, M.-B., Korth, A., Klecker, B., and Balogh, A.: Cluster observations of the exterior cusp and its surrounding boundaries under northward IMF, Geophys. Res. Lett., 29, 56-60, 2002.

Lavraud, B., Phan, T. D., Dunlop, M. W., Taylor, M. G. G. T., Cargill, P. J., Bosqued, J.-M., Dandouras, I., Rème, H., Sauvaud, J.-A., Escoubet, C. P., Balogh, A., and Fazakerley, A.: The exterior cusp and its boundary with the magnetosheath: Cluster multi-event analysis, Ann. Geophys., 22, 3039-3054, 2004,

SRef-ID: 1432-0576/ag/2004-22-3039.

Le, G., Blanco-Cano, X., Russell, C. T., Zhou, X.-W., Mozer, F., Trattner, K. J., Fuselier, S. A., and Anderson, B. J.: Electromagnetic ion cyclotron waves in the high altitude cusp: Polar observations, J. Geophys. Res., 19 067-19080, 2001.

Le Queau, D. and Roux, A.: Heating of oxygen ions by resonant absorption of Alfvén waves in a multicomponent plasma, J. Geophys. Res., 97, 14 929-14 946, 1992.

Nykyri, K., Cargill, P. J., Lucek, E. A., Horbury, T. S., Balogh, A., Lavraud, B., Dandouras, I., and Rème, H.: Ion cyclotron waves in the high altitude cusp: CLUSTER observations at varying spacecraft separations, Geophys. Res. Lett., 30, 12-1, 2003.

Onsager, T. G. and Fuselier, S. A.: The location of magnetopause reconnection for northward and southward interplanetary magnetic field, Geophys. Monogr. Ser., 84, 183-197, 1994.

Paschmann, G., Haerendel, G., Sckopke, N., Rosenbauer, H., and Hedgecock, P. C.: Plasma and magnetic field characteristics of the distant polar cusp near local noon - The entry layer, J. Geophys. Res., 81, 2883-2899, 1976.

Pickett, J. S., Franz, J. R., Scudder, J. D., Menietti, J. D., Gurnett, D. A., Hospodarsky, G. B., Braunger, R. M., Kintner, P. M., and Kurrth, W. S.: Plasma waves observed in the cusp turbulent boundary layer: An analysis of high time resolution wave and particle measurements from the Polar spacecraft, J. Geophys. Res., 19 081-19 100, 2001.

Pinçon, J. L. and Lefeuvre, F.: Local characterization of homogeneous turbulence in a space plasma from simultaneous measurements of field components at several points in space, J. Geophys. Res., 96, 1789-1802, 1991.

Pinçon, J. L. and Motschmann, U.: Multispacecraft filtering:general framework, in Analysis methods for multi-spacecraft data, Int. Space Sci. Inst., Bern, Switzerland, 65-78, 1998.

Rème, H., Aoustin, C., Bosqued, J. M., Dandouras, I., Lavraud, B., Sauvaud, J. A., Barthe, A., Bouyssou, J., Camus, Th., CoeurJoly, O., Cros, A., Cuvilo, J., Ducay, F., Garbarowitz, Y., Medale, J. L., Penou, E., Perrier, H., Romefort, D., Rouzaud, J., Vallat, C., Alcaydé, D., Jacquey, C., Mazelle, C., dUston, C., Möbius, E., Kistler, L. M., Crocker, K., Granoff, M., Mouikis, C., Popecki, M., Vosbury, M., Klecker, B., Hovestadt, D., Kucharek, H., Kuenneth, E., Paschmann, G., Scholer, M., Sckopke, N., Seidenschwang, E., Carlson, C. W., Curtis, D. W., Ingraham, C., Lin, R. P., McFadden, J. P., Parks, G. K., Phan, T., Formisano, V., Amata, E., Bavassano-Cattaneo, M. B., Baldetti, P., Bruno, R., Chionchio, G., Di Lellis, A., Marcucci, M. F., Pallocchia, G., Korth, A., Daly, P. W., Graeve, B., Rosenbauer, H., Vasyliunas, V., McCarthy, M., Wilber, M., Eliasson, L., Lundin, R., Olsen, S., Shelley, E. G., Fuselier, S., Ghielmetti, A. G., Lennartsson, W., Escoubet, C. P., Balsiger, H., Friedel, R., Cao, J.-B., Kovrazhkin, R. A., Papamastorakis, I., Pellat, R., Scudder, J., and Sonnerup, B.: First multispacecraft ion measurements in and near the Earths magnetosphere with the identical Cluster ion spectrometry (CIS) 
experiment, Ann. Geophys., 19, 1303-1354, 2001,

\section{SRef-ID: 1432-0576/ag/2001-19-1303.}

Rönnmark, K.: Kiruna Geophysical Institute Report, 179, 1982.

Russell, C. T.: The Polar Cusp, Adv. Space Res., 25, 1413-1424, 2000.

Sahraoui, F., Pinçon, J. L., Belmont, G., Rezeau, L., CornilleauWehrlin, N., Robert, P., Mellul, L., Bosqued, J. M., Balogh, A., Canu, P., and Chanteur, G.: ULF wave identification in the magnetosheath: The k-filtering technique applied to Cluster II data, J. Geophys. Res., 108, 1-1, 2003.

Sahraoui, F., Belmont, G., Pinçon, J. L., Rezeau, L., Balogh, A. P. R., and Cornilleau-Wehrlin, N.: Magnetic turbulent spectra in the magnetosheath: new insights, Ann. Geophys., 22, 22832288, 2004,

SRef-ID: 1432-0576/ag/2004-22-2283.

Smith, M. F. and Lockwood, M.: Earth's magnetospheric cusps, Rev. Geophys., 34, 233-260, 1996.
Sundkvist, D., Vaivads, A., André, M., Wahlund, J.-E., Hobara, Y., Joko, S., Krasnoselskikh, V. V., Bogdanova, Y. V., Buchert, S. C., Cornilleau-Wehrlin, N., Fazakerley, A., Hall, J.-O., Rème, H., and Stenberg, G.: Multi-spacecraft determination of wave characteristics near the proton gyrofrequency in high-altitude cusp, Ann. Geophys., 23, 983-995, 2005,

SRef-ID: 1432-0576/ag/2005-23-983

Tsurutani, B. T., Lakhina, G. S., Ho, C. M., Arballo, J. K., Galvan, C., Boonsiriseth, A., Pickett, J. S., Gurnett, D. A., Peterson, W. K., and Thorne, R. M.: Broadband plasma waves observed in the polar cap boundary layer: Polar, J. Geophys. Res., 103, 17 351-17366, 1998.

Tsyganenko, N. A.: A magnetospheric magnetic field model with a warped tail current sheet, Planet. Space Sci., 37, 5-20, 1989.

Vontrat-Reberac, A., Bosqued, J. M., Taylor, M. G. G. T., Lavraud, B., Fontaine, D., Dunlop, M. W., Laakso, H., CornilleauWerhlin, N., Canu, P., and Fazakerley, A.: Cluster observations of the high-altitude cusp for northward interplanetary magnetic field: A case study, J. Geophys. Res. (Space Physics), 108, 8-1, 2003. 